\title{
ESTRUCTURAS ORGANIZATIVAS EN EMPRESAS DE INTERNACIONALIZACIÓN ACELERADA: EVIDENCIA EMPÍRICA
}

\author{
FEDERICO QUESADA CHAVES \\ Escuela de Ciencias de la Administración \\ Universidad Estatal a Distancia, Costa Rica \\ fequesada@uned.ac.cr \\ JOSÉ PLA BARBER \\ Doctorado en Dirección de Empresas \\ Universidad de Valencia \\ Jose.Pla@uv.es
}

\section{RESUMEN}

Las pequeñas y medianas empresas internacionales pueden clasificarse en tradicionales, y empresas de internacionalización acelerada o born global. A pesar de que existe una variedad importante de investigaciones en el tema, ninguna ha establecido si existen diferencias entre la organización formal de una empresa de internacionalización acelerada y una empresa internacional tradicional. Mediante un estudio de casos a nivel comparativo, se establecen dos empresas internacionales tradicionales en la industria turística y de alimentos, y se analizan sus estructuras formales, con respecto a dos empresas en las mismas industrias con características de born global. Se determinan diferencias en los temas de socialización, centralización, flexibilidad, lateralidad y mecanismos de control.

PALABRAS CLAVE: PEQUEÑA Y MEDIANA EMPRESA, INTERNACIONALIZACIÓN, ORGANIZACIÓN, FLEXIBILIDADY ESTRUCTURA.

\begin{abstract}
Small and medium international enterprises (SMES) can be either classified as traditionally international or as enterprises with accelerated internationalization processes known as "Born Globals." Regardless of an important variety of research on the subject, the existence of differences between the formal organization of a Born Global enterprise and a traditional international enterprise has not been established. Using a case study
\end{abstract}

methodology, this article compares the formal structures of two traditional international SMEs from the tourist and food industries, and two born global enterprises from the same industries. Differences are found in features such as socialization, centralization, flexibility, laterality and control mechanisms.

KEYWORDS: SMALL AND MEDIUM ENTERPRISES, INTERNATIONALIZATION, ORGANIZATION, FLEXIBILITY AND STRUCTURE.

\section{INTRODUCCIÓN}

El objetivo de esta investigación es determinar si efectivamente las empresas Born Global tienen diferencias organizativas importantes con respecto a las pequeñas y medianas empresas internacionales tradicionales. La metodología que seguirá esta investigación utilizará como marco teórico, y punto de partida al trabajo realizado por Gabrielsson, Kirpalany, Dimitratos, Solber y Zuchella (2008), ya que es la única investigación que ha logrado homologar los procesos de internacionalización de pequeñas y medianas empresas tradicionales y los procesos de internacionalización de las Born Global, y adicionalmente, ha encontrado similitudes y diferencias entre ambos. Es con esta propuesta que se plantea la siguiente pregunta de investigación: ¿Existen diferencias significativas entre la estructura organizativa que tiene una pequeña y mediana empresa que sigue un proceso de internacionalización tradicional, y una empresa Born Global o de internacionalización acelerada? 
Este tipo de investigación resulta particularmente relevante, ya que las empresas de internacionalización acelerada son un fenómeno producto de la globalización (Madsen y Servais, 1997), y aunque ya han sido investigadas, todavía se desconocen algunos elementos de su funcionamiento. Conocer el tipo de estructura organizativa de empresas born global, y cómo se diferencia de sus contrapartes más tradicionales, puede proporcionar herramientas analíticas a los formuladores de política económica en países altamente expuestos al mercado mundial; donde es más probable que se presente este fenómeno, tal es el caso de Costa Rica. Un estudio de esta naturaleza también puede contribuir facilitando los procesos de acreditación a nivel internacional, proporcionando un antecedente.

\section{MARCO TEÓRICO}

La Born Global es considerada una pequeña y mediana empresa con un comportamiento altamente innovador, si se concibe la internacionalización como un proceso de innovación (Knight y Cavusgil, 2004). El comportamiento organizativo de las pequeñas y medianas empresas dependerá del contexto en el cual se desenvuelvan. Sin embargo, existen condiciones básicas en las que los estudiosos de la estrategia y estructura en pequeñas medianas empresas concuerdan, los cuales serán explicados en la siguiente sección.

Según la indagación realizada, solo se pudo encontrar un documento que ha diferenciado los procesos de internacionalización que sigue una empresa pequeña o mediana internacional tradicional, de una empresa Born Global (Gabrielsson et al., 2008) y que a la vez ha proporcionado algún conocimiento sobre cuáles pueden ser las posibles cualidades organizativas producto de la formación de estrategias para Born Globals. Esta investigación será utilizada como parámetro desde una perspectiva teórica para guiar los esfuerzos de esta investigación.

\section{Modelos de internacionalización acelerada o no lineal}

Antes de iniciar esta sección, es importante realizar algunas aclaraciones. De ahora en adelante, los siguientes términos serán considerados como sinónimos: Born Global (Madsen y Servais, 1997), Internacional New Venture (INVs) (Oviatt y Macdougall, 1994), Global start ups, y empresas de internacionalización acelerada. En segundo lugar, cuando en este documento se discutan la distribución de tareas a lo interno de una empresa, las estructuras jerárquicas, las cadenas de mando y los sistemas de recompensas que se aplican a lo interno de la empresa, se estará haciendo referencia a la estructura organizativa de esta.

Las empresas Born Global son consideradas como entidades fundadas con el objetivo de incursionar en mercados internacionales desde su creación (Oviatt y Macdougall, 1994; Oviatt y Macodugall, 1995). Esta condición propia de algunas empresas puede ser considerada como una clasificación teórica que se realiza a partir de la publicación del artículo Oviatt y Macdougall (1994), y posteriormente la fusión entre dos campos de estudio; el emprendedurismo y los negocios internacionales (Oviatt y Macdougall, 2000). Dicha unión dio lugar a lo que estos académicos llaman "el emprendedurismo internacional".

Antes de la publicación del artículo de Oviatt y Macdougall (1994), la investigación científica elaborada con el objetivo de determinar las condiciones bajo las cuales se internacionaliza una pequeña y mediana empresa podía ser clasificada en dos grandes grupos:

- Un primer grupo de académicos cuyo enfoque teórico se basa en las propuestas de innovación del producto planteadas originalmente por Rogers (1962) y Vernom (1966), y cuyas investigaciones se han concentrado en empresas norteamericanas. Según esta propuesta, cuando una empresa decide internacionalizarse, está optando por diver- 
sificar su producto para que este se adapte al mercado internacional, por lo cual, el proceso de internacionalización, es observado y analizado como una diversificación del producto en sí misma.

- Un segundo grupo de investigaciones se originaron de las propuestas teóricas del que se denominara "enfoque escandinavo" o modelo de Upsala (Pla-Barber y EscribaEstevé, 2005) debido a que ha sido asociado a los autores del norte de Europa que lo formularon (Johanson y Wiedersheim-Paul 1975; Johanson y Vahlne, 1977, 1990). El desarrollo teórico de este enfoque se sustenta en la necesidad de evadir riesgos y de realizar incursiones internacionales exitosas basadas en la eliminación de la incertidumbre; objetivo que se alcanza mediante el acceso a la información; la cual es recabada mediante un proceso de aprendizaje gerencial que permite conocer los mercados externos (Johanson y Wiedersheim-Paul, 1975). El proceso de internacionalización, según la escuela escandinava, comprende tres etapas: una primera en la que la firma realiza exportaciones indirectas, una segunda en la cual la firma exporta a través de agentes independientes, una tercera en la cual las empresas exportan a través de subsidiarias, y una cuarta etapa, cuando la empresa inicia manufacturación en el exterior (Pla-Barber y Escriba-Estevé, 2005).

Las dos corrientes de pensamiento anteriormente citadas son cuestionadas en por Oviatt y Macdougall (1994), quienes argumentan que existen empresas que una vez fundadas, en un periodo relativamente corto; unos dos o tres años posteriores a su fundación, inician un proceso de exportación con productos o mercados diversificados. Lo más importante es que ambos autores no encuentran evidencia empírica del cumplimiento de ninguna de las etapas propuestas por ambas corrientes, más bien deter- minan que muchas empresas se internacionalizan de manera acelerada y no lineal, a pesar de no contar con recursos financieros o materiales importantes.

Es importante anotar que se pueden identificar dos fenómenos que influyen sobre la internacionalización de una empresa; aquellos relacionados con el ambiente que arrastran a la empresa hacia los mercados internacionales, entre los que se puede mencionar el avance tecnológico y la alta especialización a nivel mundial. Por otro lado, también se debe citar el efecto empuje que sufre la empresa por alguna condición local inherente a la misma, tal como el achicamiento del mercado doméstico y la subsiguiente búsqueda de oportunidades en el exterior, y los efectos que tiene una gerencia agresiva y proactiva en búsqueda de oportunidades internacionales.

Este último efecto empujón ha sido ampliamente estudiado en la literatura de las Born Global (Madsen y Servais, 1997; Coviello y Munro, 1997; Pla y Escriba, 2005; Aspelund, Madsen y Moen, 2006; Freeman, Edwards y Schroder, 2006; Zhou, Wei-ping y Xueming, 2007; Gabrielsson et al., 2008), ya que se ha podido identificar un nuevo tipo de gerente más agresivo y arriesgado en la búsqueda de oportunidades en el exterior. A este comportamiento gerencial Ganinstky (2001) lo denomina policéntrico, y es la capacidad que tiene un gerente para identificar oportunidades de negocios en cualquier parte del mundo, debido a la experiencia adquirida previamente o a la formación que haya recibido. Este comportamiento por parte de los gerentes es proactivo y agresivo (Knight y Cavusgil, 2004; Autio, 2005), y en muchas ocasiones tiene una alta incidencia sobre el comportamiento organizativo que tiene la empresa, como se podrá analizar más adelante.

Oviatt y Macdogall (1994) son los primeros en proponer una clasificación con respecto al tipo de estrategia seguida por una empresa de inter- 
nacionalización acelerada basándose principalmente en la cantidad de mercados atendidos a través de la cadena de valor. A continuación se ofrece una explicación simplificada de cada una de este tipo de empresas:

- Nuevas empresas internacionales creadoras de mercados (INV, exportadora/importadora e INV comercializadora mundial): tanto la empresa exportadora/importadora como la INV comercializadora mundial, tienden a absorber las actividades organizativas de sistemas y conocimiento relacionadas con las actividades principalmente logísticas, mientras la organización de otras actividades relacionadas con otros temas son externalizadas.

- INV Focalizada: a diferencia de las empresas anteriormente mencionadas, este tipo de empresas tiene como objetivo enfocarse en mercados geográficos específicos y las ventajas competitivas provienen de la coordinación de múltiples actividades en la cadena de valor. En el caso particular de este tipo de empresas, la ventaja competitiva es mucho más difícil de imitar, ya que proviene de recursos únicos como el desarrollo tecnológico, recursos humanos y procesos de producción particulares, los cuales son producto de interacciones sociales complejas que generan un conocimiento tácito en muchas ocasiones, único.

- INV de alcance global o Global Star-up: básicamente, este tipo de entidad abarca una combinación de los dos tipos de empresas anteriormente descritos. La Global Star-up, es una empresa que obtiene ventajas competitivas tanto de la coordinación de actividades económicas a través de las cadenas de valor, como de la atención de distintos mercados geográficos diversos. Este tipo de empresa se beneficia de los mercados globalizados y obtiene recursos en cualquier parte del mundo y logra venderlos donde cuentan con más valor.

\section{Estructura organizativa de la Born Global: aprendizaje organizativo e interacción en redes}

La estructura organizativa resultante de una Born Global se encuentra definida por el entorno en el que interactúa y por las decisiones que se toman a lo interno de la empresa. La investigación de los tipos de estructura organizativa que tiene la Born Global es todavía un campo abierto (Gabrielsson et al., 2004), particularmente la distribución entre tareas, la interacción entre departamentos y los tipos de rutinas organizativas que siguen estas empresas para la generación de innovación continua.

Una empresa que interactúa en redes cuenta con una estructura particularmente diseñada para este propósito; tiene una estructura organizativa formal orientada a interactuar en un mundo donde el conocimiento compartido se vuelve una necesidad para sobrevivir y desarrollar ventajas competitivas. Snow, Miles y Coleman (1992) definen este tipo de empresas mediante la interacción que se mantenga con el ambiente (ver figura 1).

En este tipo de estructuras organizativas, se mantiene una estructura competitiva debido a que existe un encadenamiento hacia atrás y hacia delante en la cadena de valor. Mientras que en la red estable existe una importante consolidación en la red social, en la cual se desenvuelve la empresa a través de la cadena de valor, en las empresas que interactúan en redes dinámicas hay una constante mutación en búsqueda de la competitividad.

Las empresas que se organizan en red tienen una importante inclinación hacia la competitividad a nivel global, así lo establecen Snow et al. (1992), quienes argumentan que las principales razones por las cuales una empresa se organiza de esta manera son las siguientes:

- Búsqueda global de oportunidades y recursos. 
FIGURA 1

\section{Ejemplos de Red Estable y dinámica}

\section{RED ESTABLE}

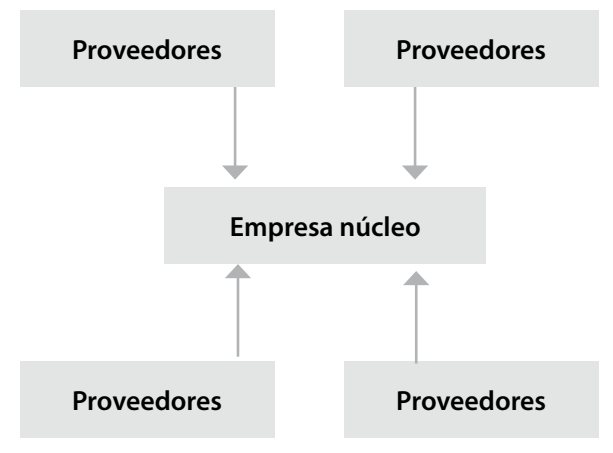

Fuente: Snow et al. (1992).

- Maximización de utilidades provenientes de activos dedicados a los negocios, ya sean propiedad de la empresa o de otras empresas.

- Desempeño solo de aquellas funciones para las cuales la empresa tiene o puede desarrollar algún tipo de habilidad especial.

- Ubicar fuera de la empresa todas aquellas actividades que pueden ser realizadas por otros agentes o empresas de una mejor manera.

Según Snow et al. (1992), si una empresa desea permanecer en el ambiente competitivo propiciado por los procesos de globalización que han surgido en los últimos 25 años, es necesario que adopten este tipo de estructuras. Según Cuervo (2001), este tipo de empresas poseen las siguientes características:

- Surge del desarrollo de la subcontratación. Consiste en un núcleo ejecutivo que contrata en el exterior el resto de actividades, tanto productivas, comerciales como incluso de I+D.

\section{RED DINÁMICA}

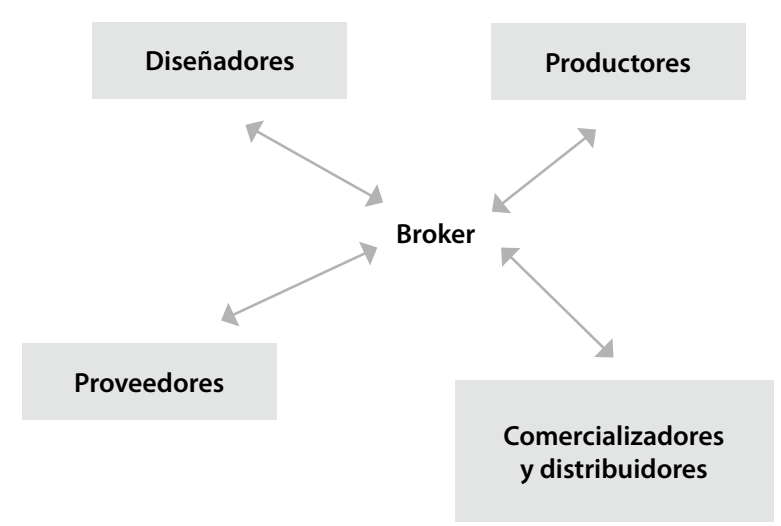

- Solo existe el ápice estratégico, hay centralización y desaparecen las variables de coordinación e integración.

- Deben crear una red estable con proveedores y clientes.

- $\quad$ No se puede considerar una estructura formal.

- Ventajas: la elevada flexibilidad.

- Inconvenientes: altos costes de contratación.

Rasgos organizativos de una empresa Born Global como el núcleo de una red

La organización que tiene una Born Global cuenta con ciertas variables organizativas formales, que la distinguen como empresa de una organización internacional tradicional, ya sea pequeña, mediana o multinacional. Las variables organizativas que se analizarán son las siguientes y la justificación de su presencia en una Born Global se exponen a continuación: 
- Aprendizaje organizativo: esta variable presente en las empresas Born Global se entiende como la posibilidad que tiene una empresa de generar y transmitir conocimiento, el cual se traduce en rutinas organizativas que a la larga generan competitividad (Catalone, Cavusgil y Zhao, 2002). Las Born Global poseen condiciones que les permiten generar conocimiento de manera constante (Knight y Cavusgil, 2004; Aspelund et al., 2006, Zhang y Tansuhaj, 2007; Gabrielsson, 2008), por lo tanto pueden innovar de manera sostenida para atender los nichos de mercado en los cuales enfocan su producción (Bell, 1995; Shrader, 2001 Ganinstky, 2001; Moen, 2002; Rialp, 2005; Freeman et al., 2006; Fenhaber et al., 2007).

- Socialización: comprendida como un imaginario colectivo que existe a lo interno de una empresa, son los hábitos, costumbres y valores que tiene esta entidad en el desempeño de sus actividades pero que no tiene un carácter formal (Pla y León, 2004). La generación de normas, y su adopción dependerá en gran medida de los hábitos que tiene la empresa para internalizar estas actividades. En general, se comprende, que la transmisión de conocimiento es necesaria para que exista un constante proceso de innovación. A este proceso de transmisión de conocimiento intra-organizacional Moornan y Miner (1998) lo determinan como las creencias colectivas y las rutinas conductuales que se encuentran relacionadas con la difusión de conocimiento entre las diferentes unidades de la empresa.

- Centralización: existen tres razones básicas para suponer que la Born Global es una organización con un alto grado de centralización en comparación con empresas que no tienen esta particularidad. Primero, según Cuervo (2001), una empresa que interactúa en redes tiene un núcleo altamente centralizado el cual funciona como un broker. Segundo, existe una importante cantidad de estudios que aseguran que el gerente de la empresa, en algunas ocasiones es también el fundador y cuenta con un comportamiento policéntrico (Madsen y Servais, 1997; Sharma y Bloomstermo, 2003; Rialp, 2005; Pla y Escribá, 2005; Freeman et al., 2006; Coviello, 2006; Zhou et al., 2007; Gabrielsson et al., 2008; Rialp et al., 2009;). Como ya se discutió anteriormente, este comportamiento es la capacidad que tiene un gerente para percibir oportunidades en diferentes partes del mundo, y la capacidad de asumirlas de una manera proactiva y agresiva (Ganinstky, 1989). Según Zhou et al. (2007), generalmente el fundador cuenta con un capital social que se transfiere a la empresa, lo cual le proporciona protagonismo a lo interno de esta. Por otro lado, cuando las empresas multinacionales que interactúan en una red interna, están realizando una incursión en un mercado exterior a través de una sucursal, la cual es reducida en tamaño, dimensiones y conocimiento, generalmente existe una amplia centralización. Conforme se amplían las operaciones y la empresa se expande, esta se descentraliza (Doz y Prahalad, 1981). Tercero, cuando se lleva a cabo el proceso de internacionalización, existe un alto empoderamiento por parte del gerente (Freeman et al., 2006), en gran parte porque el mismo pone en juego su reputación, prestigio y en algunos casos recursos económicos en la vía, por lo tanto, las decisiones tienden a concentrarse en este individuo.

- Lateralidad: la lateralidad se refiere a la interacción y envolvimiento que tienen los departamentos de la empresa en un proyecto en particular. Es por esta razón que generalmente, estas empresas, tienden a organizarse en estructuras matriciales o por proyectos (Joyce, Mcgee y Slocum, 1997); en ocasiones a través de los departamentos que confor- 
man la empresa, o a veces a través de los diferentes eslabones de la red social en la cual está interactuando la empresa. Generalmente, estas empresas tienen estas estructuras formales porque les permiten generar una mayor cantidad de conocimiento, rasgo que se puede identificar en la mayoría de las Born Global y característica necesaria para sobrevivir a entornos turbulentos (Ibarra, 1993).

- Flexibilidad: entendida como la capacidad que tiene una empresa para adaptarse a su entorno aunque este tenga un elevado nivel de incertidumbre. Esta capacidad se encuentra presente en las Born Globals (Madsen y Servais, 1997; Rialp, 2005; Zhou et al., 2007). La flexibilidad le permite a la empresa adaptar todas aquellas innovaciones generadas en la empresa producto de la generación del conocimiento, y que, por lo tanto, pueden ser aprovechadas en la producción de bienes y servicios directamente comerciables a nivel internacional.

\section{El proceso de Internacionalización de la pequeña y mediana empresa: Born Globals y pymes tradicionales, diferencias y semejanzas}

Comentar sobre el proceso de internacionalización que sigue este tipo de empresas puede volverse bastante complejo, ya que existen varias investigaciones que se han centrado específicamente en investigar la organización de la empresa para facilitar la incursión internacional, e inclusive existe una agenda para determinar cuáles industrias son más propensas a internacionalizarse (Fernhaber et al., 2007). Esta consulta atenderá básicamente el proceso de internacionalización comparado entre pymes tradicionales y Born Globals (Gabrielsson et al., 2008). Esta última investigación se utilizará como marco de referencia para el proceso de internacionalización que sigue la Born Global en comparación con la pequeña y mediana empresa internacional tradicional (Figura 2).

FIGURA 2

\section{Secuencias del proceso de internacionalización de una pequeña y mediana empresa tradicional y una Born Global}

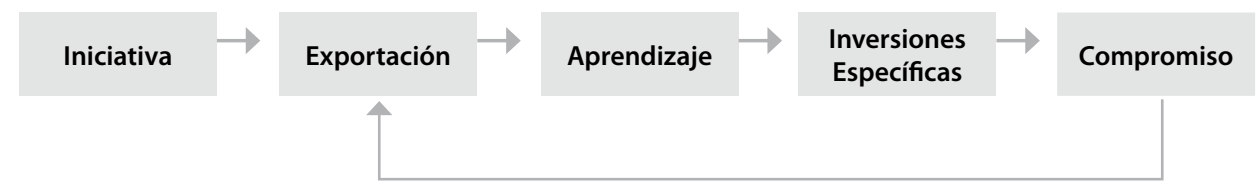

Proceso tradicional de Internacionalización de una pequeña y mediana empresa

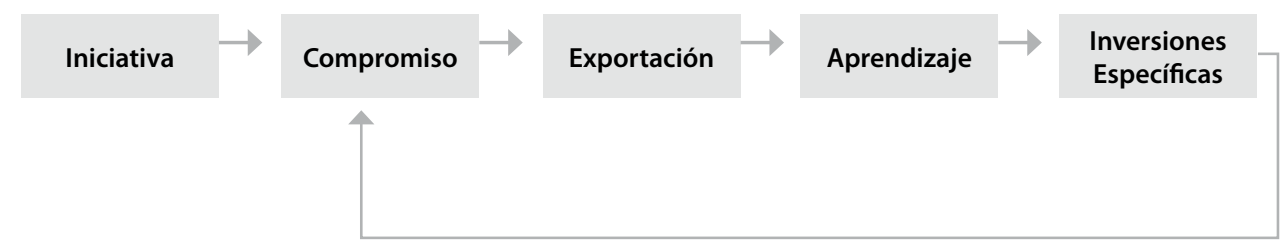

Proceso de internacionalización de una Born Global

Fuente: Gabrielsson et al. (2008). 
Este modelo puede considerarse una recopilación de otras investigaciones que han utilizado el paradigma de los recursos y capacidades para indagar acerca del modelo de internacionalización que siguen las Born Globals. A continuación se ofrece una explicación de cada una de las etapas de este modelo:

- La iniciativa corresponde al estímulo con visión global que recibe el fundador de la empresa, que en algunas ocasiones proviene de su antecedente empresarial.

- El compromiso corresponde a una variable no directamente medible, la cual delimita a un fundador que tiene la seguridad que su empresa será líder, este concepto aparece en la investigación realizada por Coviello (1997).

- Las exportaciones son inmediatas, ya que las redes y las relaciones sociales que tiene el fundador se lo facilitan para la Born Global, sin embargo para que la pequeña y mediana empresa tradicional, el proceso es un aprendizaje lento dependiendo de la estrategia que esta asuma hacia el proceso de internacionalización.

- El aprendizaje organizacional ocurre a través de las relaciones sociales mantenidas, las cuales se materializan por medio de contactos formales e informales, a través de los cuales las empresas aprenden mediante el acceso a socios ya sean grandes (para aquellas empresas que decidan adherirse en redes a socios comerciales grandes) o de cualquier otro tipo.

- Las inversiones específicas corresponden al despliegue de recursos que hace la empresa internacional, el cual debe ocurrir antes de que la empresa inicie el proceso de aprendizaje organizativo. En algunos casos se acude a capital de riesgo.

En el modelo original propuesto por Mika Gabrielsson, V.H. Marek Kirpalani, Pavlos Dimitratos, Carl Arthur Solberg y Antonella Zuchella, se reconocen tres etapas de internacionalización para las empresas Born Globals: una primera etapa, cuyo objetivo es introducirse en el mercado internacional, una segunda etapa, cuya meta es la acumulación de recursos financieros y materiales para acelerar el crecimiento, y finalmente, una tercera etapa en la cual la empresa adquiere una estrategia global la cual se deriva de todo el conocimiento organizativo y la experiencia acumulada de consumidores que demandan productos a nivel global. A continuación se exponen brevemente cada una de estas tres etapas y sus consecuencias para la empresa.

\section{Primera fase: Introducción}

y fase inicial de lanzamiento

La creación de una estrategia de negocios será determinada por el tipo de red que adopte la empresa para llevar a cabo el proyecto empresarial. Según Gabrielsson et al. (2008), si la empresa decide implementar una estrategia mediante un padrino' que ya cuenta con experiencia en los mercados internacionales, es muy probable que esta tenga un crecimiento dramático de una manera más rápida, ya que se superan algunos de los obstáculos financieros que enfrenta la mayoría de las empresas pequeñas y medianas internacionales que buscan oportunidades en el exterior (Gabrielsson et al., 2004). Por otro lado, si la empresa decide construir su demanda individual utilizando canales de distribución y redes informales en su mayoría, es muy probable que el proceso de internacionalización tome más tiempo.

\section{Segunda Fase: Crecimiento y acumulación de recursos}

En un segundo momento, cuando la empresa ya alcanza cierto nivel de consolidación, se genera una acumulación de recursos que le permitirá a

1. Estos autores reconocen en un padrino, a una empresa multinacional ya consolidada que facilite el proceso de internacionalización a través de apoyo ya sea mediante referencias, apoyo logístico o financiero. 
esta mantenerse en el mercado internacional. La recopilación de estos recursos será determinada mayoritariamente por dos variables: la capacidad de la empresa para convertirse en una industria global y la capacidad que tiene para enfrentar la globalización. En estos momentos las empresas deben implementar un aprendizaje organizativo exhaustivo de sus clientes y socios comerciales con los cuales interactúa, esto con el objetivo de determinar cuáles serán las experiencias que le permitirán planear una estrategia global en la siguiente fase.

En este momento particular en el tiempo, la empresa no enfrenta una competencia importante en el mercado internacional debido a lo particular que resulta su producto, por lo tanto, el éxito de la empresa vendrá determinado en su mayoría, por la capacidad que tenga esta para establecerse en el mercado y para seleccionar los canales y redes correctos de distribución.

\section{Tercera fase: Selección de nuevo camino o nuevo mercado}

Finalmente, en este punto la empresa ya cuenta con un bagaje de conocimiento organizacional, que se ha adquirido durante las fases previas de empresas que interactúan en la cadena de valor; tanto empresas productoras que han apoyado la creación del producto, como de consumidores a nivel global. En este momento se inicia la planificación de una estrategia global derivada de este conocimiento, la cual será implementada tomando en consideración los clientes ya consolidados o dejándolos de lado para incursionar en nuevos mercados; todo dependerá de la estrategia seleccionada. Para determinar cuál será la estrategia que seguirá la empresa Born Global en esta fase del camino, los autores que formularon este modelo señalan que existen cuatro vectores que determinarán la estrategia que seguirá la empresa los cuales se exponen a continuación y tendrán relevancia, tanto si la empresa decide involucrarse en una red global conformada por un padri- no o empresa multinacional ya consolidada, o si la estructura de la red se encuentra dispersa y la empresa ha decidido participar en esta:

1. Hasta qué punto se puede abandonar independencia y convertirse en un pequeño satélite de la red global

2. Hasta qué punto se puede aceptar cerrar el monitoreo de todas sus acciones

3. Hasta qué punto se puede aceptar ser simplemente un contratista afiliado

4. Deberá buscar los recursos necesarios y las competencias necesarias para buscar una estrategia de quiebre, con lo cual se liberaría parcial o totalmente de una empresa que ha sido su padrino.

\section{METODOLOGÍA}

Variables para clasificar empresas internacionales

El parámetro dominante en esta investigación, será el desarrollado por Oviatt y Macdougall (1994), es a partir de esta propuesta que se presentan las siguientes variables para determinar si una empresa es Born Global; considerando las particularidades de esta investigación, una cantidad específica de variables para empresas Born Global que comercializan productos en los mercados internacionales y una cantidad específica de variables para empresas que se dedican a la comercialización de servicios competitivos a nivel internacional, haciendo hincapié en empresas turísticas Born Globals (Agndal y Elbe, 2007):

- Visión internacional desde antes de la fundación de la empresa por parte de la gerencia, con una alta tolerancia al riesgo.

- Empresa concebida desde el principio para realizar transacciones internacionales o para el mercado interno.

- Periodo entre la fundación de la empresa y la primera exportación realizada. 
- Características del producto exportado.

- Globalidad (cantidad de mercados en los cuales se realizaron incursiones y diferentes tipos de productos con los cuales se ingresó).

- Uso de redes para facilitar la incursión en mercados internacionales.

Desde una perspectiva de los recursos y las capacidades, las empresas turísticas cuentan con ciertas particularidades que las distinguen de empresas exportadoras tradicionales. La diferencia más marcada entre una empresa exportadora de productos o servicios de índole tradicional, y una empresa turística, es que la segunda debe importar a los consumidores debido a que en la mayoría de los casos, la ventaja competitiva proviene de recursos que se encuentran ubicados geográficamente en un lugar específico (Bjorkman y Kock, 1997). La internacionalización en empresas turísticas es un tema poco estudiado (Bjorkman y Kock, 1997; Agndal y Elbe, 2007) y la convención, tanto para empresas que siguen un proceso de internacionalización tradicional cuyas teorías explicativas provienen de la escuela de Upsala (Johanson y Wiedersheim-Paul 1975; Johanson y Vahlne, $1977,1990)$ y de la teoría del ciclo del producto (Vernom, 1966), no aplican para explicar cómo se internacionalizan este tipo de empresas. Otro problema inherente a la internacionalización de empresas turísticas, es la naturaleza del producto ofrecido el cual no puede ser evaluado de una manera directa por el cliente, con lo cual, la información suministrada por la empresa resulta vital para poder proporcionar el servicio. Sin ánimo de intentar agotar un tema aún más incipiente que las Born Globals: las Born Globals turísticas (Agndal y Elbe, 2007). Para los objetivos de esta investigación, se considerarán Born Globals turísticas, todas aquellas empresas que se fundan con el objetivo de proveer información y servicios turísticos, a posibles turistas extranjeros.

\section{Las empresas internacionales y su estructura organizativa}

La necesidad de desarrollar más investigación con respecto a las características organizativas concretas de empresas Born Globals ya ha sido mencionada en otros trabajos de investigación relativos al tema (Gabrilesson et al., 2004). La conformación de departamentos y su interacción en este tipo de empresas, tiene una relación más compleja que sus contrapartes tradicionales. Esto se debe a que la empresa gestiona un conocimiento adquirido, mientras que en otro tipo de empresas internacionales (multinacionales) su desarrollo se da en una amplia y compleja red de relaciones sociales (Bartlett y Ghoshal, 1990). Básicamente, una empresa Born Global, concentra, procesa, analiza y genera una gran cantidad de información con respecto a los mercados internacionales; actividades que una empresa multinacional le exigen una red global de relaciones sociales y una generación de valores, rutinas organizativas y desarrollo de procesos y procedimientos con un uso extensivo de recursos financieros, humanos y materiales. Hasta ahora no existe una convención con respecto a qué tipo de organización formal tiene una empresa Born Global, más bien existen algunas reglas prácticas con respecto a las características organizativas que tienen estas empresas (Knight y Cavusgil, 2004 y 2005; Zhang y Tansuhaj, 2007; Zhou et al. 2007).

Quizá uno de los hallazgos más importantes con respecto a las Born Globals, se refiere a la capacidad que tienen para generar estrategias internacionales exitosas, tomando en consideración ciertos recursos clave (Oviatt y Macdougall, 1994). A partir de estos recursos, siguiendo el paradigma de Chandler (1962), se genera un tipo de estructura que permite alcanzar esta estrategia, lo cual impacta sobre la organización de la firma. Según algunos de los hallazgos más importantes, y a partir de los cuales se genera esta investigación, las Born Globals se organizan a partir de la infraestructura tecnológica (Zhang 
y Tansuhaj, 2007), de las orientaciones a mercadearse en el exterior, hacia la generación de conocimiento (Knight y Cavusgil, 2004 y 2005), y a partir de las redes sociales que tiene el gerente de la empresa, el cual en algunos casos es el fundador de la misma (Zhou et al., 2007).

A partir de la literatura revisada, lo que se intentará es obtener información con respecto a la generación de las rutinas organizativas que tienen las empresas analizadas. Se dará una respuesta a lo siguiente: establecer cuáles son las rutinas organizativas que tienen las cuatro empresas internacionales que se estudiarán, cuáles son las estrategias que implementan, y cómo se ajustan las estructuras organizativas de las empresas para cumplir con estas estrategias. A continuación se ofrece una mención de cada una de las variables que se estudiarán para determinar las diferencias que existen entre una empresa internacional tradicional, perteneciente a la industria manufacturera y posteriormente, se exponen las variables que serán analizadas para determinar las diferencias en las estructuras que tiene una empresa internacional de servicios dedicados al turismo, y una empresa internacional tradicional dedicada al turismo:

- Centralización.

- Innovación y aprendizaje organizativo.

- Lateralidad.

- Socialización.

- Flexibilidad.

- Mecanismos de control.

\section{Selección de objeto de estudio y recopilación de información}

Para la selección del objeto de estudio de esta investigación, se procedió a indagar diferentes bases de datos existentes en Costa Rica, así como investigaciones previamente realizadas, especialmente en el área de las PYMES. Como se mencionó al inicio del apartado de la metodología, se investigaron distintos tipos de empresas internacionales. A continuación se proporciona un breve resumen de cómo se contactó a estas empresas y los criterios que fueron utilizados para su respectiva selección.

La primera empresa internacional contactada debía cumplir con los siguientes criterios: ser en principio una empresa Born Global de manufactura, contar por lo menos con dos continentes a los cuales se estén realizando exportaciones y tener accesibilidad de información. La empresa seleccionada tiene como nombre Alimentos Don Mariano, y se encarga de producir alimentos tipo SNACKs a base de yuca y plátano frito. Esta empresa ya había sido estudiada en el proyecto "Information and communication Technologies in SMEs"2 el cual tenía como objetivo identificar empresas con un alto componente tecnológico que estuvieran realizando exportaciones a diferentes partes del mundo. La investigación existente permitió no solo analizar información correspondiente al tipo de organización que tiene la empresa en un momento determinado del tiempo, sino que también mediante un cruce de los datos obtenidos en la investigación anterior y la información adquirida en ese momento, se pudo constatar la evolución de la estructura a lo largo del tiempo.

La segunda empresa seleccionada debía cumplir con los siguientes criterios: ser en principio una empresa con mayor antigüedad que Alimentos Don Mariano, tener algún grado de internacionalización y pertenecer a la industria de bebidas y alimentos. Mediante una revisión de investigaciones anteriormente efectuadas, y un

2. Este proyecto fue contratado por la Universidad de las Naciones Unidas en el año 2004 al Instituto Latinoamericano de Políticas Públicas. El objetivo de esta investigación era identificar empresas que en ese momento estuvieran utilizando de manera exhaustiva tecnologías de la información y las comunicaciones. Esta empresa fue seleccionada por el potencial que presentaba en ese momento para convertirse en una empresa consolidada a nivel nacional e internacional. 
contacto con la Cámara de Industrias Alimentarias (CACIA), se pudo constatar que la empresa seleccionada nunca había sido investigada anteriormente. La empresa seleccionada fue Fideos Precocidos de Costa Rica, una industria que produce para el mercado nacional y también exporta pastas especialmente diseñadas para la elaboración de un plato tradicional chino-costarricense, el CHOP SUEY.

Para el segundo análisis comparativo se procedió a contactar empresas que pertenecieran a la industria turística costarricense; un sector que actualmente es muy dinámico y cuenta con una amplia proyección. Mediante diversos contactos, se pudo establecer una conexión con una industria comercializadora llamada COMTUR.S.A. Esta compañía es una comercializadora de productos turísticos, y mantiene contacto con diferentes tipos de empresas que se encuentran en el mismo sector. A través de esta empresa se pudo contactar a dos empresas de la industria hotelera. Los criterios de selección para la primera empresa fueron los siguientes: ser por naturaleza una Born Global de servicios de hotel. Según el estudio realizado por Agndal y Elbe (2007), se pudo constatar que una Born Global con estas condiciones, tiene como objetivo buscar clientes en diferentes partes del mundo, sin importar que tan remotas sean, y segundo utilizan una amplia red de intermediarios para atraer a estos clientes. La empresa que cumplió con estos fines, fue un hotel Ilamado Los Lagos Hotel, Spa y Resort, ubicado en el altamente dinámico sector turístico de La Fortuna de San Carlos. Este hotel ubicado en las faldas del Volcán Arenal, es una empresa de tipo familiar y cuenta con una amplia y compleja infraestructura de alto valor agregado, que le permite proporcionar distintos tipos de servicios tanto a turistas nacionales como extranjeros. Sin embargo, debido a lo exótica que resulta su ubicación, los turistas extranjeros lo encuentran sumamente atractivo.
Para realizar comparaciones con esta empresa, se decidió contactar a una empresa turística ya consolidada llamada Condovac La Costa. Esta empresa es una corporación cuyo objetivo es la venta de tiempo compartido en la zona turística de Guanacaste. Esta empresa tiene más de treinta años de estar operando y mantiene amplias relaciones con diferentes empresas comercializadoras de servicios turísticos. Existen algunas diferencias en el tipo de servicios que ofrecen cada una de las empresas comparadas, mientras que los servicios que ofrece Los Lagos Hotel, son de hospedaje, Condovac La Costa brinda tanto servicios hoteleros, como de venta de acciones para acceder al lugar, pero este tema será discutido más adelante. Por ahora es importante mencionar que Condovac es una empresa internacional, sin embargo no cuenta con un mercado tan desarrollado y amplio a nivel externo, como Los Lagos Hotel. Las relaciones que se espera observar entre las cuatro empresas observadas se encuentran fundamentadas en la propuesta originalmente planteada por Grabrielsson et al. (2008), con lo cual se espera obtener información sobre la internacionalización.

Para finalizar esta sección, se indica que la información obtenida de ambas empresas, fue recopilada mediante el uso de entrevistas semiestructuradas realizadas en las organizaciones objeto de análisis. Las entrevistas realizadas contaron con dos secciones; una primera cuyo objetivo fue analizar en qué condiciones se fundó la empresa, cuáles fueron las motivaciones y experiencias en mercados internacionales. Esta primera sección permitió realizar una clasificación de la empresa, para determinar si el proceso de internacionalización que siguió fue tradicional o de tipo acelerado, y segundo poder determinar si la estructura con la que cuenta la organización le permitió atender mercados internacionales desde un principio. En la segunda serie de preguntas de la entrevista, se consultaron temas organizativos de la empresa tales como 
FIGURA 3

\section{Esquema analítico entre las diferentes empresas analizadas}

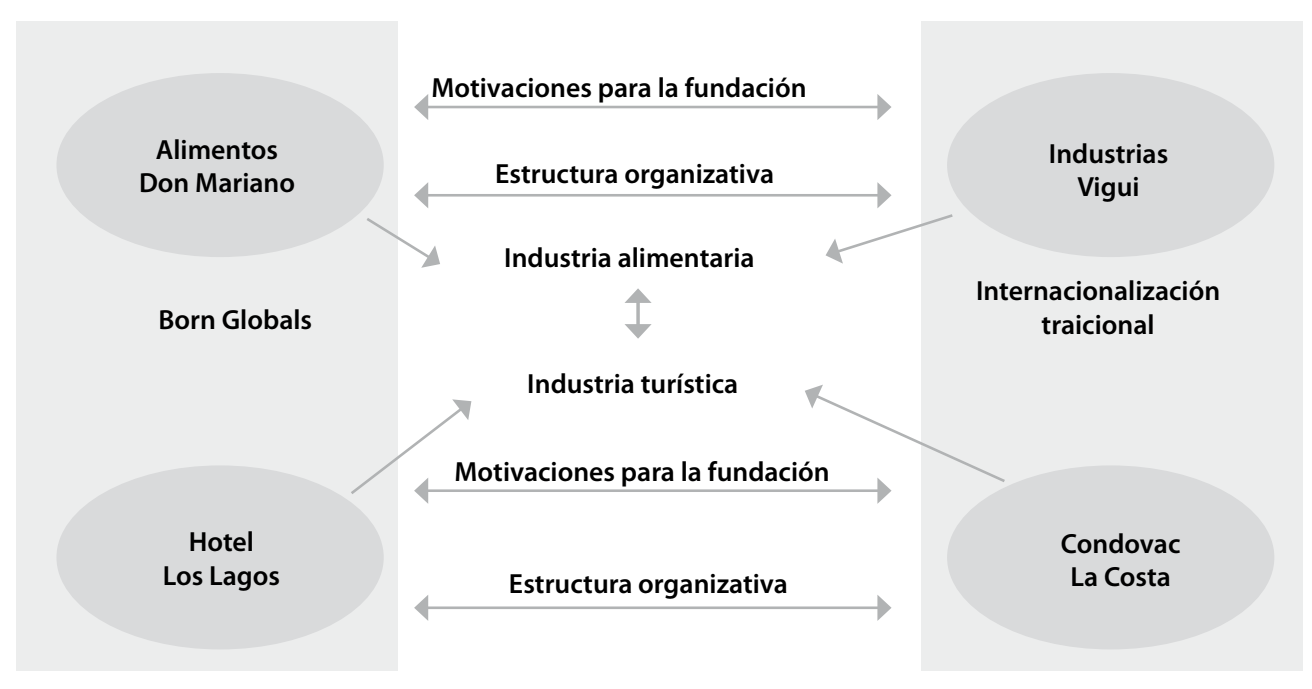

Fuente: elaboración propia.

la distribución de tareas, los sistemas de incentivos y recompensas, los procesos de socialización y centralización, y si existe o no, lateralidad. Es importante indicar adicionalmente, que por pertenecer las empresas comparadas a sectores distintos, las características de la industria fueron analizadas con el objetivo de determinar si esto influye sobre la conformación de la Born Global. Finalmente, es importante mencionar que se siguió la metodología de estudios de caso con el objetivo de generar nueva teoría a partir de los datos obtenidos.

\section{Estudios de Caso (Evidencia Empírica)}

La búsqueda de empresas Born Global, para satisfacer las necesidades empíricas de esta investigación, fue una tarea amplia y compleja. En la sección anterior se mencionaron las cuatro organizaciones analizadas y sus respectivas características. Los hallazgos fueron concluyentes; confirmaron muchas afirmaciones de la literatura y adicionalmente proporcionaron nuevas relaciones teóricas que las indagaciones previas no han mostrado. A pesar de que los hallazgos de esta investigación no se pueden generalizar, sí se plantea de una manera concluyente, que es necesario realizar una investigación de tipo cuantitativo, para determinar si algunos de los hallazgos obtenidos en esta investigación son propios de las empresas que se internacionalizan de manera más acelerada, o son producto de la ubicación geográfica, cultura o condiciones industriales en las cuales se han desarrollado.

Cada estudio de caso se realizó con el objetivo de determinar, primero, la duración, el grado y la intensidad del crecimiento internacional, y segundo, todas aquellas variables organizativas que pudieran dar indicios de cómo se organiza la empresa de manera formal. Entre los hallazgos más relevantes, se encuentra la presencia de una alta centralización en torno a las figuras de la gerencia general en las empresas que han tenido una internacionalización más temprana e intensa, así como la presencia de estructuras más flexibles en este tipo de empresas. A continuación se ofrece un breve resumen de los hallazgos más relevantes para esta investigación. 
RNA Revista Nacional de Administración

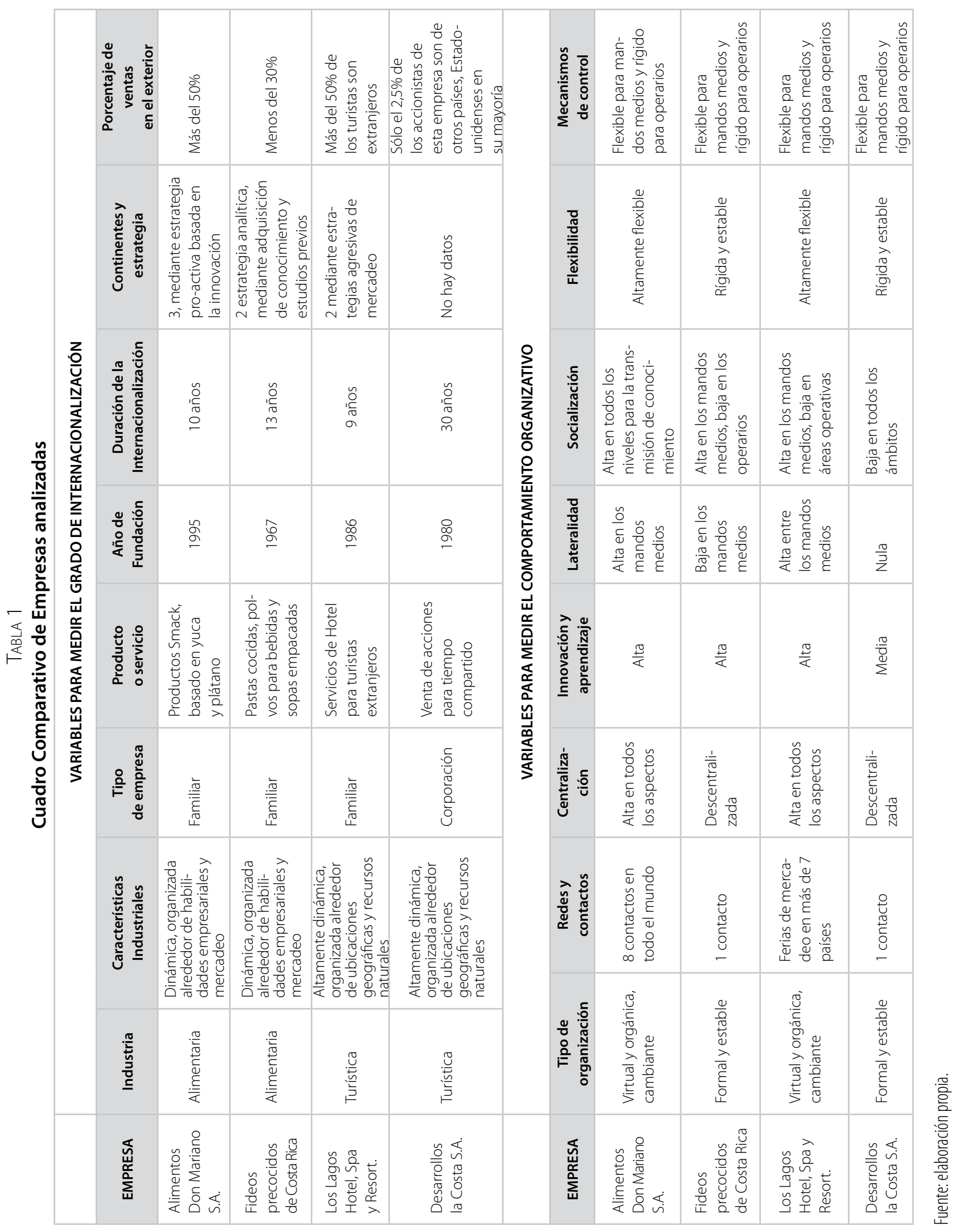




\section{Resultados}

A partir del trabajo de Gabrielsson et al. (2008) y otros trabajos adicionales (Coviello y Munro, 1997; Coviello, 2006; Coviello y Cox, 2007), se derivaron algunos comportamientos organizativos especiales que tienen las empresas que se internacionalizan de manera no lineal. Las empresas de internacionalización acelerada crecen gracias a la cantidad y el tipo de contactos con los que cuentan, es decir, hacen un uso exhaustivo de las redes sociales en las que interactúan. Esta interacción, transforma el capital social de los gerentes o empleados clave de la empresa, en capital social de toda la empresa (Zhou et al., 2007), y es a partir de este comportamiento que la empresa se organiza de una manera particular, que la diferencia de una PYME que haya crecido en el exterior de manera tradicional y de una transnacional.

En un mayor o menor grado, este tipo de redes determina cómo se organiza la empresa, para el aprovechamiento de sus recursos productivos que la hacen competitiva en el exterior. El trabajo empírico realizado en esta investigación, abarcó un análisis comparativo entre dos tipos de industrias en Costa Rica; la industria alimentaria, y la industria turística. Para la primera industria se escogió una empresa que presentara rasgos similares a una Born Global, y una empresa que diera indicios de haberse internacionalizado de una manera tradicional. El mismo ejercicio se realizó para la industria turística; y se centró la atención y los esfuerzos en dos empresas; una con características de Born Global Turística (Agndal y Elbe, 2007) y una PYME turística tradicional.

Se alcanzaron las siguientes conclusiones con respecto al comportamiento en el exterior de las empresas en la industria alimentaria: mientras que Alimentos Don Mariano, no puede ser clasificada como una Born Global de alcance global debido a que tardó diez años en internacionalizarse, sí puede ser clasificada como una Born Global, porque cumple con dos criterios adicio- nales, exporta más del $50 \%$ de su producto, y ha ingresado en tres continentes en un periodo relativamente corto, por lo tanto se clasificó como una Born Global comercializadora mundial, la cual tiene la capacidad especial de crear un mercado donde antes no existía.

Por otro lado, Fideos Precocidos de Costa Rica, realizó su primera exportación 13 años después de su fundación y actualmente solo exporta un $30 \%$ de su producto, en gran parte porque es una empresa altamente precavida que solo exporta después de realizar estudios de mercado y adquirir conocimiento del lugar en el exterior donde planea exportar. Con respecto al tipo de redes y contactos observados en ambas empresas, se pudo constatar que Alimentos Don Mariano cuenta con más de seis contactos a nivel internacional, mientras que Fideos Precocidos tan solo cuenta con uno.

Con respecto a la Industria Turística, se determinó lo siguiente con respecto al comportamiento internacional de las empresas analizadas: Hotel Los Lagos, Spa y Resort es sumamente agresiva en términos de mercadeo en el exterior, y se internacionalizó en un lapso de nueve años, por lo tanto se clasificó como una Born Global turística (Agndal y Elbe, 2007). Adicionalmente, se comprobó que al igual que Alimentos Don Mariano, construyó toda su red de contactos a nivel nacional, y en el exterior, lo cual muy probablemente incidió sobre el tiempo que le tomó internacionalizarse. Con respecto a Desarrollos La Costa S.A., se pudo concluir que es una empresa que cuenta con más de 5000 accionistas, y que de estos, tan solo el 2,5\% son de otros países, lo cual se puede deber en gran parte, a que sus estrategias de mercadeo son agresivas en el mercado local, mas no así en el mercado exterior. A continuación se exponen los resultados obtenidos de la investigación:

- Flexibilidad: estructura formal en red, flexible para una Born Global para poder adaptarse rápidamente a las necesidades de sus 
clientes e interactuar de manera más ágil con respecto a su entorno, estructura formal rígida para una PYME tradicional. Se determinó que las empresas que se habían internacionalizado de manera lineal, tendían a tener una estructura formal y rígida, mientras que las empresas Born Globals son sumamente flexibles y se adaptan para responder a las demandas de clientes internacionales y a cambios en el entorno. Esto se reflejó en el cambio de la estructura, la cual resultaba altamente flexible para responder a esta realidad.

- Centralización: alta centralización en la Born Global, descentralización en la PYMES tradicional. Se pudo comprobar que las empresas Born Global tendían a encontrarse altamente centralizadas en torno a la figura del gerente, quien en gran medida era, al mismo tiempo, el fundador de la empresa en ambos casos. Por otro lado, se pudo constatar que las PYME internacionales tradicionales tendían a estar relativamente descentralizadas.

- Orientación hacia el aprendizaje organizativo: alto aprendizaje organizativo en la Born Global, debido a la necesidad de sobrevivir en el exterior; moderado aprendizaje organizativo en la PYME internacional tradicional, ya que esta última primero se afianza totalmente en el mercado que está atendiendo. Esta variable mostró resultados mixtos, mientras que las empresas Born Global, mostraron tendencias moderadas hacia el aprendizaje organizativo, ya que la mayoría del planteamiento de nuevos proyectos se encuentra limitado a las propuestas de la gerencia, y los mandos medios en ambas empresas. Ambas PYMEs tradicionales, mostraron una tendencia hacia el aprendizaje organizativo, especialmente Fideos Precocidos de Costa Rica, la cual centra su atención en el mejoramiento continuo de procesos y la innovación.
- Orientación hacia la innovación: la Born Global innova de una manera más rápida que la PYME internacional tradicional. Ambas Born Globals mostraron una tendencia a la innovación, ya que las dos han creado una mayor cantidad de productos y servicios en un periodo más corto, así como también se han preocupado de que estas innovaciones se adapten al mercado internacional. Por otro lado, las PYMES tradicionales, mostraron una tendencia moderada a la innovación, primero Fideos Precocidos de Costa Rica, con su incursión en productos para bebidas, y Desarrollos La Costa, con la construcción de nuevas y más completas instalaciones, sin embargo, ambas empresas continúan mercadeando su producto de una manera más tradicional.

- Socialización: existe un proceso de socialización más importante en la Born Global debido a la necesidad de monitorear más mercados, y de generar una mayor dinámica innovadora. Hay una baja o moderada socialización en las PYMES internacionales tradicionales, ya que no existe una necesidad explícita de gestionar conocimiento e innovación de una manera dinámica. Se pudo constatar que la socialización del conocimiento en ambas Born Globals, se presenta en los mandos medios, y esta no trasciende a las secciones operativas de la empresa, lo cual es, en gran parte, producto de la centralización de la toma de decisiones. Por otra parte, se pudo constatar que la socialización en las PYMES tradicionales tiende a ser moderada en los mandos medios y baja en las secciones operativas, producto de la alta estandarización en ambas empresas.

- La lateralidad: se espera en la Born Global, la existencia de una estructura formal lateral, que facilite la socialización del conocimiento y las innovaciones. Se espera una lateralidad nula por parte de las PYMES internacio- 
nales tradicionales. Se pudo constatar que existe una importante lateralidad en ambas empresas Born Globals con el objetivo de gestionar el conocimiento y las decisiones estratégicas de la organización, sin embargo esta se presenta en los mandos medios, y no en los mandos operativos. Adicionalmente, se pudo constatar que la lateralidad, es todavía más baja en las PYMES tradicionales.

- Mecanismos de control: Se esperan mecanismos de control más estrictos para las Born Globals, debido a que el mercado internacional es más exigente. En la PYME tradicional, se esperó un comportamiento más rígido en todos los niveles debido a que este tipo de empresas cuentan con una mayor estandarización en sus procesos. Se pudo constatar que prácticamente en las cuatro empresas se presentaba exactamente la misma situación, en los mandos medios existe relativa flexibilidad y rígida en los mandos operativos. Con respecto al tipo de pago, las remuneraciones al recurso humano tienden a ser constantes en los cuatro casos.

\section{CONCLUSIONES Y FUTURAS LÍNEAS DE INVESTIGACIÓN}

De este trabajo se desprenden una serie de conclusiones relevantes para el estudio de la dirección de empresas internacionales. En general, la mayoría de los estudios acerca de las Born Globals se han centrado en analizar un concepto latente denominado capacidades organizativas, a pesar de la relevancia de realizar este tipo de indagaciones, estas no son directamente observables, como sí lo son los departamentos y las acciones llevadas a cabo por estos. La estructura formal de una empresa es directamente observable mediante sus departamentos, su interacción y los resultados que producen, y a pesar de la existencia de una gran cantidad de literatura acerca de Born Globals indagada para esta investigación, no se pudo hallar un solo documento que proporcionara una delimitación de estructura formal para estas empresas.

Esta investigación proporciona la primera estructura formal para empresas Born Global, sus características y funcionamiento. En general se puede concluir, que las empresas Born Globals cuentan con una estructura formal más flexible que sus contrapartes tradicionales, sin embargo también se encuentra altamente centralizada dado el papel protagónico que juega el gerente/fundador de la empresa; debido a esta característica básica, se vuelve necesaria una mayor capacitación en el desarrollo de habilidades policéntricas entre los empresarios.

Adicionalmente se destaca que la empresa Born Global cuenta con una estructura organizativa formal especialmente diseñada para aprender, y por lo tanto existe socialización como variable latente, la cual también es directamente observable en la estructura formal, mediante una alta lateralidad entre los departamentos. Tanto la mayor lateralidad como la socialización, dan como resultado una mayor inclinación hacia el aprendizaje organizativo; como variable latente, y esto da como resultado una mayor innovación en productos directamente observable en las empresas Born Globals.

Ningún estudio es perfecto, y este cuenta con una serie de limitaciones que se exponen a continuación: primero, las empresas analizadas como Born Globals, tuvieron que pasar un periodo de acumulación de contactos en el exterior que retardó el proceso de internacionalización, en relación con Born Globals que han contado con un padrino, por lo tanto es necesario analizar de manera agregada este segundo tipo de Born Globals para añadir robustez a este tipo de investigación. Las empresas analizadas corresponden a industrias dinámicas en Costa Rica, sin embargo es necesario analizar Born Globals de otras industrias, especialmente relacionadas con 
tecnologías de punta, ya que es en este tipo de empresas donde se puede evidenciar de mejor manera el aprendizaje organizativo. Finalmente, las empresas Born Globals de alcance global son aquellas organizaciones donde la internacionalización se presenta de una manera más agresiva; es necesario identificar cuál es el papel del gerente de la empresa y determinar si el comportamiento observado en esta investigación se puede constatar en este tipo de empresas.

Para concluir, se plantea realizar las siguientes investigaciones con el objetivo de profundizar las indagaciones de estructuras formales en las Born Globals y en empresas internacionales tradicionales. Primero realizar una investigación cuantitativa sobre la forma en que toman las decisiones las empresas Born Globals, y cómo impactan estas al resto de la estructura organizativa de la empresa. Esta investigación en gran parte se justifica por el papel que juega el gerente de la empresa, el cual según lo observado en el trabajo de campo, es el fundador y tiende a tener un protagonismo importante en todas las decisiones que toma la empresa.

Para finalizar, también se propone realizar una investigación de índole cuantitativa, en empresas Born Globals de diferentes industrias, con el objetivo de determinar el tipo de estructuras formales existentes, y las características que predominan, a fin de proporcionar sustento inductivo a la investigación deductiva realizada en este informe. Adicionalmente, es necesario realizar una investigación dinámica de las estructuras formales de las Born Globals, en un estudio de datos de panel para alcanzar resultados válidos que permitan deducir, si los datos observados con respecto a las variables organizativas analizadas en este documento pueden ser generalizados a un espacio más amplio.

\section{Referencias}

Agndal, H. y Elbe, J. (2007). The Internationalization Processes of Small and Medium-sized Swedish Tourism Firms. Scandinavian Journal of Hospitality and Tourism, 7(4), 301-307.

Aspelund, A., Madsen, T. y Moen, O. (2006). A review of the foundation, international marketing strategies, and performance of international new ventures. European Journal of Marketing, 41(11), 1423-1448.

Autio, E. (2005). Creative tension: the significance of Ben Oviatt's and Patricia McDougall's article'toward a theory of international new ventures'. Journal of International Business Studies, 36, 9-19.

Bell, J. (1995). The internationalization of small computer software firms: A further challenge to "stage" theories. European Journal of Management, 29(8), 60-75.

Bjorkman, I. y Kock, S. (1997) Inward international activities in service firms - Illustrated by three cases from the tourism industry. International Journal of Service Industry Management, 8(5), 362-376.

Catalone, R., Cavusgil, S. y Zhao, Y. (2002). Learning Orientation, firm innovation capability, and firm performance. Industrial Marketing Management, 31(5), 515-524.

Chandler, A.J. (1962). Strategy and Structure: Chapters in the history of the American Industrial Enterprise. Recuperado de: Google books.google.co.cr

Coviello, N. y Munro, H. (1997). Network Relationships and the Internationalisation Process of Small Software Firms. International Business Review, 6(4), 361-386.

Coviello, N. (2005). Integrating qualitative and quatitative techniques in network analisys. Qualitative market research, 8(1), 39-60.

Coviello, N. (2006). The network dynamics of international new ventures. Journal of International Business Studies, $37,713-731$.

Coviello, N. y Cox, M. (2007). The resource dynamics of international new venture networks. Journal of International Entrepreneurship, 4, 113-132.

Cuervo, A. (2001). Introducción a la administración de empresas. Madrid: Cívitas.

Desarrollos La Costa S.A. (2010). Condovac La Costa S.A. Recuperado de www.condovac.com 
Dimitratos, P., Johnson, J., Slow, J. y Young, S (2003). 'Micromultinationals: New Types of Firms for the Global Competitive Landscape, European Management Journal, 21(2), 164-174.

Doz, Y. y Prahalad, C. K. (1981). Patterns of strategic control within multinacional corporations. Sloan Management Review, 23(1), 15-29.

Fideos Precocidos de Costa Rica S.A. (2010). Productos, Tecnología y Salud. Recuperado de www.vigui.com

Freeman, S. y Cavusgil, S.T. (2007). Toward a Typology of Commitment States Among Managers of Born-Global Firms: A Study of Accelerated Internationalization. Journal of International Marketing, 15(4), 1-21.

Freeman, S., Edwards, R. y Schroder, B. (2006). How Small Globals use networks and Alliances to overcome constraints to rapid internationalization. Journal of International Business Studies, 14(3): 33-63.

Gabrielsson, M., Kirpalany, V., Dimitratos, P., Solber, C. y Zuchella, A. (2008). Born Globals: Propositions to help advance the theory. International Business Review, 17, 385-401.

Gabrielsson, M., Sassi, V. y Dharling, J. (2004). Finance Strategies of rapidly growing Finish SMES: Born Internationals and Born Globals. European Business Review, 16(6), 590-604.

Ganitsky, J. (1989). Strategies for innate adoptive exporters: lessons from Israel s case. International Marketing Review, 6(5), 50-65.

Ibarra, H. (1993). Network Centrality, power, and innovation involvement: Determinants of technical and administrative roles. Academy of Management Journal, 36(3), 471-591.

Instituto Costarricense de Turismo (2009). Anuario Estadístico 2009. Recuperado de http://www.visitcostarica. com/ict/paginas/ict.asp

Johanson, J. y Vahlne, J.E. (1977). The internationalisation process of the firm - a model of knowledge development and increasing foreign market commitment. Journal of International Business Studies, 8, 23-32.

Johanson, J. y Vahlne, J.E. (1990). The mechanism of internationalization. International Marketing Review, 7(4), $11-34$.
Johanson, J. y Wiedershei-Paul F. (1975). The Internationalization of the Firm: Four Swedish Cases. Journal of Management Studies, 305-322.

Joyce, W.; Mcgee, V.E. y Slocum, J.J. (1997). Designing Lateral Organizations: an analysis of the benefits, costs and enablers of non-hierarchical organization forms. Decision Sciences, 28(1), 1-25.

Knight, G. y Cavusgil, T. (2004). Innovation, organizational capabilities, and the born-global firm. Journal of International Business Studies, 35, 124-131.

Knight, G. y Cavusgil, T. (2005). A Taxonomy of Born-global Firms. Management International Review, 45(3), 15-35.

Knight, G.; Madsen, T.K. y Servais, P. (2004). An inquiry into Born Global firms in Europe and USA. International Marketing Review, 24(6), 645-665.

Madsen, T.K. y Servais, P. (1997). The Internationalization of Born Globals: an Evolutionary Process? International Business Review, 6(6), 561-583.

Asamblea Legislativa de la República de Costa Rica. Ley de Fortalecimiento de las PYMEs, Número 8262. Recuperado de www.pgr.go.cr

Los Lagos Hotel, Spa y Resort. (2010). Historia. Recuperado de www.hotelloslagos.com

Miles, R.E. y Snow, C.C. (1978). Organizational Strategy, Structure and Process. Academy of Management Review July, 546-564.

Ministerio de Economía, Industria y Comercio de la República de Costa Rica. (s.f). Reglamento General a la Ley No. 8262 de Fortalecimiento de las Pequeñas y Medianas Empresas. Recuperado de www.pgr.go.cr

Moen, O. (2002). THE BORN GLOBALS: A new generation of Small European Exporters. International Marketing Review, 19(2/3), 156-176.

Moornan, C. y Miner, A. (1998). Orgazational Improvisation and organizational memory. Academy of Management Review, 23(4), 698-723.

Oviatt, B.J. y Macdougall, P.P. (1994). Toward a theory of International New Ventures. Journal of International Business Studies, 25(1), 45-64.

Oviatt, B.J. y McDougall, P.P. (1995). Global start-ups: entrepreneurs on a worldwide stage. Academy of Management Executive, 9(2), 30-43. 
Oviatt, B.J. y McDougall, P.P. (2000). International Entrepreneurship: The Intersection of Two Research Paths. Academy of Management Journal 43(5), 902-906.

Pastas Roma. (2010). Pastas Roma. Recuperado de www. pastasroma.com

Pla-Barber, J. y Escribá-Esteve. (2005). Accelerated internationalization: evidence from a late investor country. International Marketing Review, 23(3), 255-278.

Rialp, A., Rialp, J. y Knight. (2005). The phenomenon of early internationalizing firms: what do we know after a decade of scientific inquiry (1993-2003)?. International Business Review, 14, 147-166.

Rialp, A., Rialp, J., Urbano, D. y Vaillant, D. (2005). The BornGlobal Phenomenon: A Comparative Case Study Research. Journal of International Entrepreneurship 3, 133-171

Rialp, A., Galvan, I. y Suarez. (2009). A configuration-holistic approach to born-global firms strategy formation process. European Management Journal: en prensa.

Rogers, E. (1962), Diffusion of Innovations, The Free Press, New York, NY.

Shrader, R.C. (2001). Collaboration and performance in foreign markets: The Case of young high-technology manufacturing firms. Academy of Management Journal, 44(1), 45-50.

Snow, M.; Miles, R. y Coleman, H. (1992). Managing 21st Century Network Organizations. Organizational Dynamics.

Sharma, D. y Blomstermo, A. (2003). The internationalization process of Born Globals: a network view. International Business Review, 12, 739-753.

Zhang, M. y Tansuhaj, P. (2007). Organizational Culture, Information Technology Capability, and Performance: The case of Born Global firms. Multinational Business Review, 3(15), 43-76.

Zhou, L.; Wei-ping W. y Xueming, L. (2007). Internationalization and the performance of born-global SMEs: the mediating role of social networks. Journal of International Business Studies, 38, 673-690.

Recibido: 29-06-2011

Aceptado: 29-10-2011 